\title{
Slurry-Based Processing of Solid Electrolytes: A Comparative Binder Study
}

\author{
Nathalie Riphaus, $\mathbb{1}^{1,2, *, z}$ Philipp Strobl, ${ }^{1,2}$ Barbara Stiaszny, ${ }^{2}$ Tatiana Zinkevich,, 3 \\ Murat Yavuz, ${ }^{3}$ Joscha Schnell, ${ }^{5}$ Sylvio Indris, $\oplus^{3,4}$ Hubert A. Gasteiger, ${ }^{1, * *}$ \\ and Stefan J. Sedlmaier $\mathbb{B}^{2}$ \\ ${ }^{1}$ Chair of Technical Electrochemistry, Department of Chemistry and Catalysis Research Center, Technische Universität \\ München, 85748 Garching, Germany \\ ${ }^{2}$ BMW Group, 80809 Munich, Germany \\ ${ }^{3}$ Institute for Applied Materials - Energy Storage Systems, Karlsruhe Institute of Technology, 76344 \\ Eggenstein-Leopoldshafen, Germany \\ ${ }^{4}$ Helmholtz Institute Ulm, 89081 Ulm, Germany \\ ${ }^{5}$ Institute for Machine Tools and Industrial Management, Technische Universität München, 85748 Garching, Germany
}

Limited energy density of today's Li-ion battery technologies demands for novel cell technologies, such as the all-solid-state battery (ASSB). In order to achieve high energy densities and enable large-scale processing, thin and flexible solid electrolyte (SE) layers have to be implemented. This study focuses on slurry-based processing of the sulfidic solid electrolyte $\mathrm{Li}_{10} \mathrm{SnP}_{2} \mathrm{~S}_{12}$ (LSPS). Various polymers were investigated concerning their suitability as binders for thin and freestanding SE sheets. We conducted a parameter study in order to optimize e.g. LSPS-to-binder ratio, solids content and porosity. Significant differences were found with regard to the minimum amount of binder required for mechanically stable sheets as well as the homogeneity, density and flexibility of the resulting SE layers. The impacts of binder type and weight fraction on ionic conductivity were examined through lithium diffusion measurements. Impedance analysis was conducted in comparison, proving sufficiently high ionic conductivity for potential application of the SE sheets in ASSB. This work highlights the important role of the polymeric binder in slurry-based processing of SEs and gives an impression how important a well-considered selection of parameters is to achieve good processing properties as well as desirable features for the final SE sheet.

(C) The Author(s) 2018. Published by ECS. This is an open access article distributed under the terms of the Creative Commons Attribution 4.0 License (CC BY, http://creativecommons.org/licenses/by/4.0/), which permits unrestricted reuse of the work in any medium, provided the original work is properly cited. [DOI: 10.1149/2.0961816jes]

Manuscript submitted October 9, 2018; revised manuscript received December 3, 2018. Published December 22, 2018. This was Paper 323 presented at the Cancun, Mexico, Meeting of the Society, September 30-October 4, 2018.

Battery electric vehicles (BEV) are constantly gaining popularity. Effective penetration of the mass market, however, requires a significant improvement in energy density whilst keeping costs reasonable. This is mainly owed to the demand for extended driving ranges above $500 \mathrm{~km}$ (300 miles). These ranges ask for volumetric energy densities exceeding $800 \mathrm{Wh} \cdot 1^{-1}$ at cell level. In contrast, only half of this value is reached in current prismatic cells of BEVs, based on established Li-ion battery (LIB) technologies. ${ }^{1}$

The all-solid-state battery (ASSB) concept has attracted growing interest as a promising battery system to potentially achieve higher energy density. ${ }^{2}$ This is based on the assumption that solid electrolytes (SE) should more likely enable the use of lithium metal as anode material as well as high-voltage cathode materials compared to liquid electrolytes, although recent studies revealed that the initially postulated high electrochemical stability was a misconception. ${ }^{3}$ Most bulk-type ASSBs reported to date are based on thick SE layers and cathodes with low cathode active material loadings, resulting in energy densities below $50 \mathrm{Wh} \cdot 1^{-1}$ at cell level. ${ }^{4,5}$ Indeed, for being competitive with conventional LIBs, energy density calculation reveal that the SE layer thickness has to be in the $<100 \mu \mathrm{m}$ range. ${ }^{6}$ In addition, a certain mechanical stability is essential for scalable processing. ${ }^{7}$ Consequently, evolution from pellet-type ASSBs, prepared by powder compression, to sheet-type ASSBs, which are based on slurry-coating processes, is essential. ${ }^{4,8}$

The fabrication of thin sheet-like layers in turn demands the use of polymeric binders that are chemically compatible with the SE materials. ${ }^{9}$ This on the other hand allows for addressing two challenges of ASSBs. One major problem is attributed to rigid solid-solid interfaces that lead to high total cell resistances. ${ }^{10,11}$ Introduction of a soft binder that enhances the flexibility of the SE layer could improve adhesion to the electrodes. Its softness might moreover reduce issues evolving from volume changes of the active materials during

\footnotetext{
*Electrochemical Society Member.

**Electrochemical Society Fellow.

${ }^{\mathrm{z}}$ E-mail: nathalie.riphaus@tum.de
}

cycling, which cause inconsistent interfaces between the SE layer and the electrodes. ${ }^{12-14}$ Due to their deformable nature, sulfide-based SEs can generally be processed easier and enable better contacting with the electrodes, compared to oxide-based SEs., ${ }^{2,3}$ Furthermore they provide higher ionic conductivities of up to $25 \mathrm{mS} \cdot \mathrm{cm}^{-1}$, exceeding those of current liquid electrolytes. ${ }^{15-17}$ Their high reactivity toward moisture, however, requires handling under inert atmosphere. $^{6}$

In general, slurry-based processing of SEs demands to carefully consider the choice of solvent and binder. Besides chemical stability with the SE material, typical processing challenges like shrinkage and warpage during drying and densification of the SE sheet need to be overcome. ${ }^{6}$ Requirements on the binder include solubility in the solvent, non-reactivity with the SE, good adhesive strength and minimal effect on the resistivity of the SE layer. ${ }^{6,18}$ Polymeric binders with polar functional groups such as nitrile, which can interact with the SE, have been reported as favorable. ${ }^{18}$ In the case of sulfide-based SEs this however contrasts with the demands on the solvent, which has to be less polar due to their high reactivity with polar protic solvents. Hence, the number of applicable binders for sulfidic SEs is limited. ${ }^{19}$

To date, literature related to solution-based processing of ASSBs is scarce, and even less is reported on fabrication of thin, freestanding SE layers. Nam et al. applied a polymer scaffold (i.e. a non-woven porous polymeric material) to obtain bendable SE layers of roughly $70 \mu \mathrm{m}$ thickness. ${ }^{20}$ They tested two sulfide-based SEs, crystalline $\mathrm{Li}_{10} \mathrm{GeP}_{2} \mathrm{~S}_{12}$ (LGPS) and glass-ceramic $\mathrm{Li}_{3} \mathrm{PS}_{4}$ (LPS). The latter has also been used in a study by Lee et al., ${ }^{18}$ who investigated different solvents and binders. They reported the combination of acrylonitrile butadiene rubber (NBR) with p-xylene, yielding SE sheets with an AC impedance derived ionic conductivity effectively equal to pressed LPS samples. It was concluded that although incorporation of a nonconductive binder is expected to reduce the ionic conductivity of the SE layer according to various reports, ${ }^{4,7,9,14,19}$ the good distribution and ion-dipole interaction of NBR results in a compensation of the intrinsically lower conductivity. The benefits of forming a freestanding SE layer instead of directly coating the SE on one of the electrodes 
became clear in a study by Ito et al. ${ }^{21}$ They found, for instance, that using the same binder in the electrode and the SE layer leads to mutual dissolution at the interface.

In the present study, we applied a slurry-coating process to fabricate bendable thin and freestanding SE sheets. The thiostannate analogue of LGPS, $\mathrm{Li}_{10} \mathrm{SnP}_{2} \mathrm{~S}_{12}$ (LSPS), that shows a comparably high ionic conductivity of $2-5 \mathrm{mS} / \mathrm{cm},{ }^{22-25}$ and toluene were selected as the model electrolyte and solvent, respectively. We systematically investigated a wide range of binders with regard to their impacts on processability, flexibility, density and resistivity of the resulting SE layer and found clear differences between the binders. For those exhibiting good processability, homogeneous distribution between SE particles, chemical compatibility with LSPS and excellent flexibility of the compressed sheets is shown. Moreover, we evaluated the impact of binder content on ionic conductivity. Compared to recent studies, ${ }^{7,18-20}$ this contribution demonstrates a significant expansion of tested binders and analytical tests, addressing important requirements for scalable roll-to-roll processing of ASSBs.

\section{Experimental}

Materials.-All materials were handled within an argon filled glove box $\left(\mathrm{O}_{2}, \mathrm{H}_{2} \mathrm{O}<1 \mathrm{ppm}\right.$; GS Glovebox Systemtechnik GmbH). $\mathrm{Li}_{10} \mathrm{SnP}_{2} \mathrm{~S}_{12}(>95 \%$, LSPS) was purchased from NEI Corporation and used without further purification. Polyisobutene (PIB) at an average molecular weight $\mathrm{M}_{\mathrm{w}}$ of $3.1 \cdot 10^{6} \mathrm{~g} \cdot \mathrm{mol}^{-1}$ was delivered by BASF SE. Poly(methyl methacrylate) (PMMA, $\mathrm{M}_{\mathrm{w}} 4-5.5 \cdot 10^{5} \mathrm{~g} \cdot \mathrm{mol}^{-1}$ ) was purchased from Alfa Aesar and poly(styrene-co-butadiene) (SBR, $\mathrm{M}_{\mathrm{w}}$ $1.9 \cdot 10^{5} \mathrm{~g} \cdot \mathrm{mol}^{-1}$ ) at a butadiene content of $4 \mathrm{wt} \%$ from Sigma Aldrich Corp. Poly(ethylene vinyl acetate) (PEVA, $\mathrm{M}_{\mathrm{w}} 3.5 \cdot 10^{5} \mathrm{~g} \cdot \mathrm{mol}^{-1}$ ) containing $60 \mathrm{wt} \%$ vinyl acetate and poly(acrylonitrile butadiene) (HNBR, $5.5 \cdot 10^{5} \mathrm{~g} \cdot \mathrm{mol}^{-1}$ ) with $17 \mathrm{wt} \%$ acetonitrile were provided by Arlanxeo. All binders were dried in a vacuum oven (Büchi B-585 Drying) at $80^{\circ} \mathrm{C}$ for 72 hours. Toluene was purchased from Merck Millipore and dried over molecular sieve (pore size $3 \AA$, Merck Millipore) before use.

Fabrication of SE sheets.-All process steps were conducted in an argon filled glove box. LSPS was first homogenized using mortar and pestle and then dispersed in toluene by stirring. The binder was dissolved in toluene, and the respective amount of binder solution was added to the LSPS dispersion. After stirring for at least 12 hours, the viscosity of the slurry was adjusted and the slurry was coated on siliconized polyester foil (PPI Adhesive Products $\mathrm{GmbH}$ ) using the doctor blade technique. The SE sheets were dried at room temperature for 1 hour at ambient pressure and further 12 hours under dynamic vacuum. For compression, the sheets were sealed into aluminum foil pouch and calendered at $60^{\circ} \mathrm{C}$ outside the glove box. The gap size was reduced by $10 \mu \mathrm{m}$ per step until the pouch bag started curling up.

Determination of porosity.-Samples were punched (Nogamigiken Co. Ltd, $12 \mathrm{~mm}$ diameter) of the SE sheet and their height and weight was measured. The porosity was then calculated as the difference between measured and theoretical density.

Scanning electron microscopy (SEM).-To assess the homogeneity, SEM and energy dispersive X-ray diffraction (EDX) mapping was conducted. The samples were therefore mounted on the sample holder and transferred to the SUPRA 55VP (Zeiss) in an air-tight transport vessel to minimize exposure to ambient air $(<5 \mathrm{~s})$.

Determination of mechanical properties.-Flexibility was evaluated with a Mandrel Bend Test according to DIN-EN-ISO 1519:2011, using cylindrical mandrels at a diameter of 8 to $3 \mathrm{~mm}$. The freestanding $\mathrm{SE}$ sheets were fixed in a self-made holder system developed at the $i w b$.

\begin{tabular}{|c|c|c|}
\hline Binder & Average $M_{w}$ & Structure \\
\hline Polyisobutene (PIB) & $3.1 \cdot 10^{6} \mathrm{~g} \cdot \mathrm{mol}^{-1}$ & \\
\hline $\begin{array}{l}\text { Styrene butadiene } \\
\text { rubber (SBR) }\end{array}$ & $1.9 \cdot 10^{5} \mathrm{~g} \cdot \mathrm{mol}^{-1}$ & \\
\hline $\begin{array}{l}\text { Poly(methyl } \\
\text { methacrylate) } \\
\text { (PMMA) }\end{array}$ & $4-5.5 \cdot 10^{5} \mathrm{~g} \cdot \mathrm{mol}^{-1}$ & \\
\hline $\begin{array}{l}\text { Poly(ethylene vinyl } \\
\text { acetate) (PEVA) }\end{array}$ & $3.5 \cdot 10^{5} \mathrm{~g} \cdot \mathrm{mol}^{-1}$ & \\
\hline $\begin{array}{l}\text { Hydrogenated nitrile } \\
\text { butadiene rubber } \\
\text { (HNBR) }\end{array}$ & $5.5 \cdot 10^{5} \mathrm{~g} \cdot \mathrm{mol}^{-1}$ & $\mathbb{N}$ \\
\hline
\end{tabular}

${ }^{7} \mathrm{Li}$ pulsed field-gradient nuclear magnetic resonance spectroscopy (PFG NMR).- - SE sheets were fixed in polypropylene tubes and placed inside air-tight NMR tubes. Experiments were conducted at a Bruker Avance $300 \mathrm{MHz}$ spectrometer operated at a ${ }^{7} \mathrm{Li}$ frequency of $116.6 \mathrm{MHz}$. The spectrometer was equipped with a Diff50 probe, which produces pulsed field gradients of up to $30 \mathrm{~T} / \mathrm{m}$. A stimulatedecho pulse sequence in combination with bipolar gradients was used to observe the echo damping as a function of gradient strength. The detailed procedure has been previously described elsewhere. ${ }^{24}$

\section{Results and Discussion}

Selection of materials.- In order to conduct a comparative binder study, a suitable model solid electrolyte and solvent had to be selected in the first place. As the motivation behind this work is to pave the way for large-scale production of sheet-type SE layers, only commercially available materials were considered, limiting the choice of sulfidebased SEs. LSPS from NEI Corporation was selected due to its high ionic conductivity of $2-5 \mathrm{mS} \cdot \mathrm{cm}^{-1},{ }^{22-24}$ which comes close to that of its thiogermanate analogue LGPS that has been reported to have one of the highest conductivities of $12 \mathrm{mS} \cdot \mathrm{cm}^{-1} \cdot{ }^{26}$ In contrast, the price can be significantly reduced by the replacement of Ge with $\mathrm{Sn}$, making LSPS more attractive for large-scale applications. ${ }^{22}$

As mentioned, the high reactivity of sulfide-based SEs with polar protic solvents, leading to evolution of $\mathrm{H}_{2} \mathrm{~S}$, requires the use of less or non-polar solvents instead. ${ }^{19}$ Yamamoto et al. found that solvents with a high donor number, like propylene carbonate or diglyme, react with the chemically similar electrolyte LPS by nucleophilic attack. ${ }^{9}$ In contrast, various studies have shown excellent stability in solvents with low donor number, such as heptane, toluene, $p$-xylene or dichloroethane. ${ }^{5,9,18}$ Since too fast evaporation of the solvent leads to inhomogeneous coatings with bulged surfaces, a further demand for slurry-processing is a low vapor pressure. ${ }^{5}$ Therefore, toluene with a moderate vapor pressure of $3.8 \mathrm{kPa}^{27}$ was selected in this study.

Once the solvent was defined, the next step comprised the selection of binders to be tested. The baseline requirements to be met were solubility in toluene and absence of protic functional groups, which would cause again decomposition reactions with the SE. Table I shows 
Table II. Value ranges for all varied parameters of the slurry processing study.

LSPS: binder

ratio $[\mathrm{wt} \%]$

$97.5: 2.5$

$95.0: 5.0$

$92.5: 7.5$

$90.0: 10.0$
Solids content

LSPS 20-30

Binder 5-20

Overall 20-45 in the slurry [wt $\%]$

Applicator gap $[\mu \mathrm{m}]$

200-400

the chosen binders. In order to gain a comprehensive understanding of the effect of the chemical nature of the binder on its performance, diverse material classes ranging from aliphatic to aromatic, including different functional groups, were covered. PIB is a fully saturated hydrocarbon, whereas SBR contains aromatic units. PMMA and PEVA both have ester side chains attached to the hydrocarbon backbone, with the number of functional groups being much larger for PMMA. NBR provides a nitrile functionality. The hydrogenated variant HNBR was used to further differentiate from SBR.

As the binders shall be used in the SE separator layer, another key requirement is negligible electronic conductivity. To probe this, pure binder films with dry-film thicknesses of 15-30 $\mu \mathrm{m}$ were casted and tested electrochemically. Linear sweep voltammetry (LSV) was performed to apply a distinct voltage to the sample and measure the current response. While PIB, SBR and PMMA showed no current flow over the voltage range of $0-5 \mathrm{~V}$ (Figure S1a), a low current of up to $45 \mathrm{nA}$ was detected for PEVA and HNBR at elevated temperatures (Figure S1b). However, the electronic conductivity in the range of $10^{-11} \mathrm{~S} \cdot \mathrm{cm}^{-1}$, calculated from chronoamperometry measurements, is negligible. In addition, as no current peaks were detected in the LSV measurements, electrochemical stability in the applied voltage range can be concluded for all binders.

In the next step, their chemical inertness with LSPS was investigated. To provoke possible decomposition reactions, SE sheets with a LSPS: binder ratio of $90: 10 \mathrm{wt} \%$ were stored for prolonged time at $60^{\circ} \mathrm{C}$. Subsequent X-ray photoelectron spectroscopy (XPS) and Fourier transform infrared spectroscopy (FTIR) measurements showed no difference between the aged samples and the pristine materials (exemplary shown for LSPS-PEVA/-PIB in Figure S2/S3). Hence, all binders fulfilled the requirements for further investigations.

Fabrication and characterization of freestanding $S E$ sheets.In order to evaluate the influences of the binder on the processability, flexibility, density and resistivity of SE thin layers, freestanding LSPS sheets were prepared by slurry-processing. All relevant process parameters, namely LSPS-to-binder ratio, solids content in the slurry, applicator gap size and compression method were varied in an extensive parameter study. The ranges of the parameters employed are listed in Table II.

A first criterion for assessing the processability of the slurry was its viscosity. The solids content in the slurry served as a measure for it. In case of a low solids content, the slurry leaks out of the applicator, whereas a slurry too viscous sticks to the applicator and cannot be processed. The study revealed that the optimum viscosity strongly depends on the type of binder used, whereas the amount of binder had less impact. The quality of PEVA-based sheets turned out to be particularly dependent on the solids content. While excellent layers were yielded at roughly $45 \%$, lower solids contents resulted in very inhomogeneous layers. The viscosity of SBR- and PMMA-based slurries, in contrast, had to be in the range of $20 \%$ for comparable results. PIB as well as HNBR could be processed best at about $40 \%$ solids content for low binder contents, however the viscosity had to be reduced if the binder content was increased.

Also relevant in this context was the wettability of the slurry. A generally good wetting behavior was expected, as both solvent and carrier foil are non-polar. Depending on the polarity of the binder, the wettability of the slurry is affected to a greater or lesser extent. For those slurries based on the most polar binder, PMMA, no homoge- neous coating could be obtained throughout the wide range of tested process parameter combinations. In all cases, the SE sheet rolled up during drying, indicating that the cohesive forces within the slurry exceed the adhesive forces between slurry and carrier foil (Figure 1c). In contrast, very homogeneous coatings were obtained for slurries comprising the non-polar binders PIB and SBR (Figures 1a/1b). The best results according to optical inspection could be achieved with PEVA and HNBR. These coatings were not only very homogeneous, but also showed well-defined edges (Figures 1d/1e). Apparently, the combination of a non-polar polymer backbone and a relatively low amount of polar functional groups enables both good cohesion in the slurry and good adhesion to the carrier foil.

Independently of the LSPS-to-binder ratio as well as the solids content in the slurry, an applicator gap size of $200 \mu \mathrm{m}$ was identified as the optimum for all binders. According to the solids contents, dryfilm thicknesses ranging between roughly $50 \mu \mathrm{m}$ for SBR and $100 \mu \mathrm{m}$ for PEVA were obtained for the uncompressed sheets. Subsequent calendering allowed for reduction of the film thickness by $55-70 \%$, while the greatest densification was achieved for the thickest sheet. The final film thicknesses after calendering varied between $20-40 \mu \mathrm{m}$.

To further evaluate the SE sheets, their homogeneity after calendering was examined in greater detail. A homogeneous sample should provide small variations in coating height and porosity over the SE layer as well as uniform distribution of the binder between SE particles. To examine the latter, EDX mapping was conducted. Exemplary elemental maps and SEM images are shown in Figure 2 for LSPS layers processed with the various binders. It should be mentioned that all sheets were fabricated under identical conditions and the same batch of SE powder was used for all experiments to ensure comparability.

Significant differences can already be observed at a low magnification for the PMMA-based sheet (Figure 2c, top), which shows a very rough surface with many agglomerates. Zooming in further reveals that, contrary to the other samples, bigger particles are not embedded in the plane but rise above, increasing the roughness (Figure 2, middle). The EDX maps revealed two different distribution pattern of the binders. SBR, PIB and PMMA show a granular domain structure, with small binder agglomerates (red) between SE particles (yellow). This is most visible for the SBR-based layer (Figure 2b). In contrast, PEVA and HNBR form a film on the SE particles, indicated by a uniform orange coloration of the EDX maps (Figures $2 \mathrm{~d} / 2 \mathrm{e}$ ). While

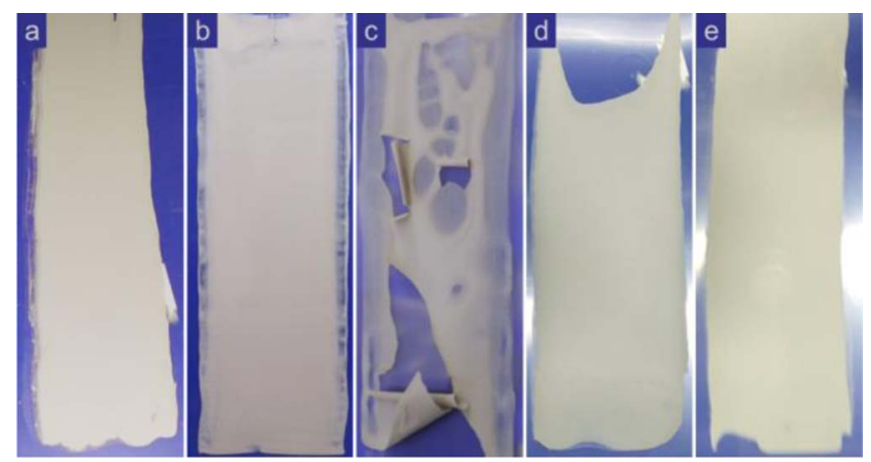

Figure 1. Images of cast SE films (200 $\mu \mathrm{m}$ wet-film thickness), containing $10 \mathrm{wt} \%$ of (a) PIB, (b) SBR, (c) PMMA, (d) PEVA and (e) HNBR 


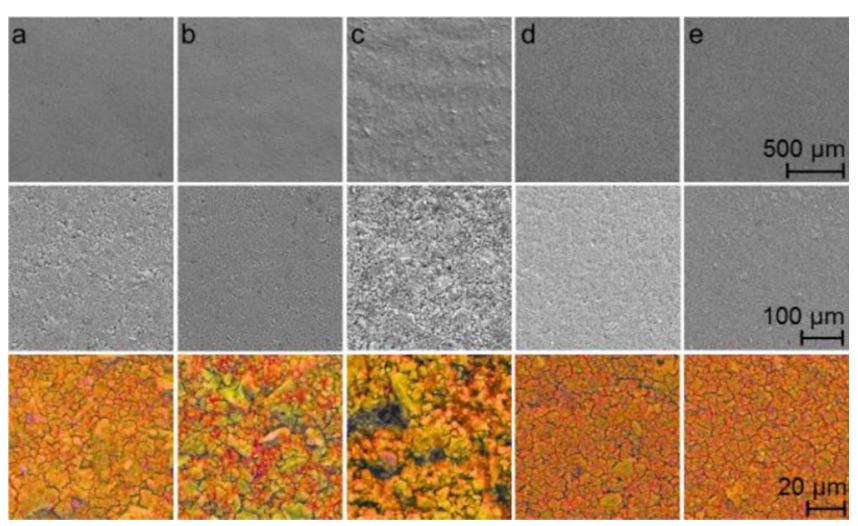

Figure 2. SEM images (top, middle) and EDX maps (bottom, yellow: sulfur, red: carbon) of SE sheets containing $10 \mathrm{wt} \%$ binder. (a) PIB, (b) SBR, (c) PMMA, (d) PEVA and (e) HNBR.

this homogeneous binder distribution apparently results in a smoother surface, the continuous film might be detrimental for the ionic conductivity. Inada et al. found that a binder coating covering the SE particles remarkably hinders ionic transport, whereas binder domains barely affect the conductivity. ${ }^{28}$ It should be noted that the gray spots arise from indentations on the surface, which cannot be reached by the EDX beam.

Both optical evaluation and SEM imaging demonstrated that PMMA is unsuitable to fabricate homogeneous and dense SE sheets, thus this binder was not further considered. Distinct differences between the remaining four were observed for the minimum amount of binder required to obtain processible, freestanding SE sheets. Previous studies have shown that the resistivity of SE layers increases with binder content, owing to the insulating behavior of the polymers. ${ }^{9,14}$ Consequently, the amount of binder should be reduced as far as possible. With SBR, $10 \mathrm{wt} \%$ (equaling to about $20 \mathrm{vol} . \%$ ) were necessary to fabricate a homogeneous, freestanding SE sheet with low porosity, whereas $7.5 \mathrm{wt} \%$ PEVA delivered comparable results. For HNBR and PIB, a much lower binder content of $2.5 \mathrm{wt} \%$ was sufficient.

These differences might be associated with the molecular weight of the polymeric binders. An interesting correlation between adhesion properties and molecular weight of the binder was reported by Lee et al., who studied LTO electrodes for conventional LIBs. ${ }^{29}$ They found that adhesion as well as surface coverage of the LTO particles enhances with increasing molecular weight of their carboxymethyl cellulose binder. The PIB used herein had the highest average molecular weight of $3.1 \cdot 10^{6} \mathrm{~g} \cdot \mathrm{mol}^{-1}$, followed by HNBR with $5.5 \cdot 10^{5}$, PEVA with $3.5 \cdot 10^{5}$ and SBR with $1.9 \cdot 10^{5} \mathrm{~g} \cdot \mathrm{mol}^{-1}$. This trend turned out to be in line with the amount of binder required for homogeneous, processible SE sheets. Apart from molecular weight, the chemical

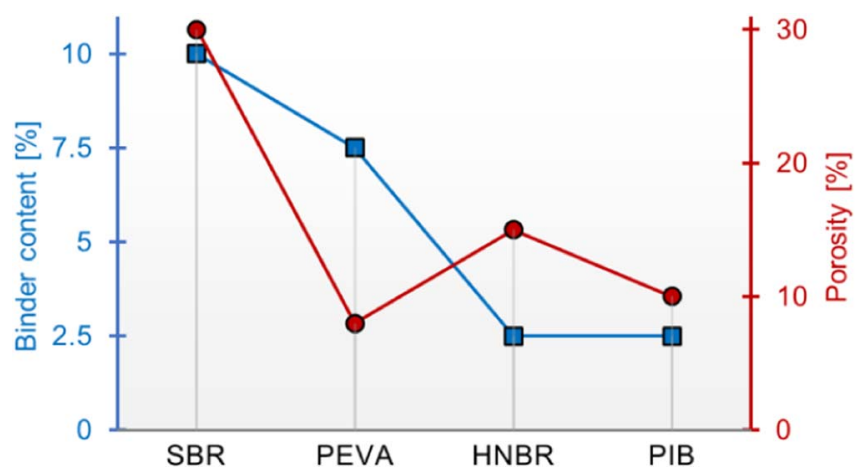

Figure 3. Relation between required minimum amount of binder to obtain freestanding, processible SE sheets (blue) and resulting porosity after calendering (red) for the different binders SBR, PEVA, HNBR and PIB.

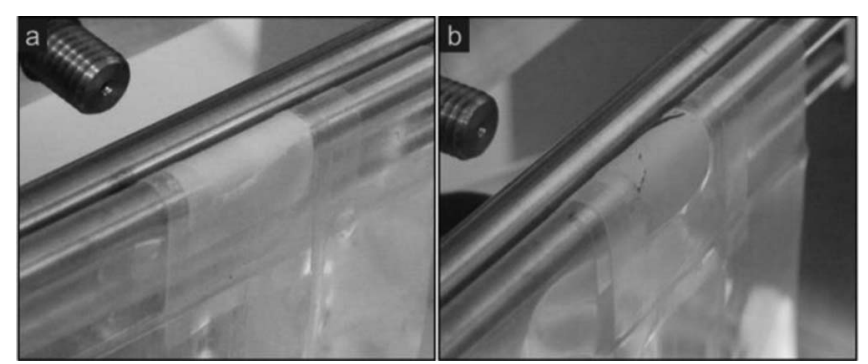

Figure 4. Mandrel Bend Tests of a calendered SE sheets with (a) $2.5 \mathrm{wt} \%$ HNBR and (b) $10 \mathrm{wt} \%$ SBR.

nature of the polymeric binder is expected to affect the properties of the SE sheet. PIB and HNBR constitute saturated hydrocarbons with small side chains, which can easily adhere to the SE particles. Lee et al. moreover found that the nitrile groups of NBR increase the polarity and dipole moment for the interaction between binder and SE, resulting in better adhesion. ${ }^{18}$ This might explain why the same binder content of $2.5 \mathrm{wt} \%$ is sufficient for PIB and HNBR, although PIB has a much higher molecular weight. PEVA and SBR contain large functional groups, which might hinder intense contact between binder and SE. Apparently, the negative effect of steric hindrance is more significant than the positive effect of polarity, thus demanding for a higher binder quantity of 7.5 and $10 \mathrm{wt} \%$, respectively.

Another important evaluation criterion is the porosity of the SE sheets after calendering. As Li-ion transport is limited with high porosity, a densification close to $100 \%$ of the theoretical density would be ideal to maximize the ionic conductivity. On the other hand, a certain remaining porosity might be beneficial to maintain good mechanical properties. $^{6,8}$ In line with this, Choi et al. found that the porosity of silicon electrodes decreases with increasing binder content. ${ }^{30}$ They concluded that a certain binder level is essential to achieve sufficient bonding between particles. Figure 3 illustrates the relation between required minimum amount of binder and resulting porosity for the various polymers. Despite the high binder content of $10 \mathrm{wt} \%$, SBRbased sheets could only be compacted to around $70 \%$ of the theoretical density upon calendering, thus roughly $30 \%$ porosity remained. In contrast, the calendered layers containing the least amount of binder, namely those with $2.5 \mathrm{wt} \% \mathrm{HNBR}$ and PIB, yielded a porosity around $15 \%$ and $10 \%$, respectively. The best results were obtained for the calendered sheets containing $7.5 \mathrm{wt} \%$ PEVA, with porosities as low as roughly $8 \%$.

These results indicate that the amount of binder has only a limiting impact on densification properties. Indeed, the chemical nature might be more relevant. The as-received polymers HNBR, and particularly PIB and PEVA, exhibited a rubberlike consistency, whereas SBR is supplied as hard granules. It is therefore not surprising that compression of the SE sheets is less effective when using SBR. The different properties can also be associated with the chemical structure of the polymer. On the one hand, the unsaturated hydrocarbon backbone introduces a certain stiffness and, on the other hand, the bulky but non-polar side chains might hinder intense bonding to the SE particles. Weaker adhesion caused by bulky functional groups could also explain why a greater amount of binder is required for PEVA compared to HNBR.

The influence of binder content and densification on the properties of the SE sheet was further examined with respect to mechanical properties. In order to assess their flexibility, Mandrel bend tests according to DIN norm ISO 1519:2011 were conducted. Calendered sheets containing 2.5 and $10 \mathrm{wt} \%$ binder as well as non-calendered sheets with $2.5 \mathrm{wt} \%$ binder were probed. All samples based on PIB, PEVA and HNBR showed excellent bending properties, even for low binder contents. They passed all mandrel diameters without any damage (Figure 4a). In contrast, all SBR-based sheets already broke at bending around the largest mandrel (Figure $4 \mathrm{~b}$ ). This observation is in line with the aforementioned poor densification properties, and can 


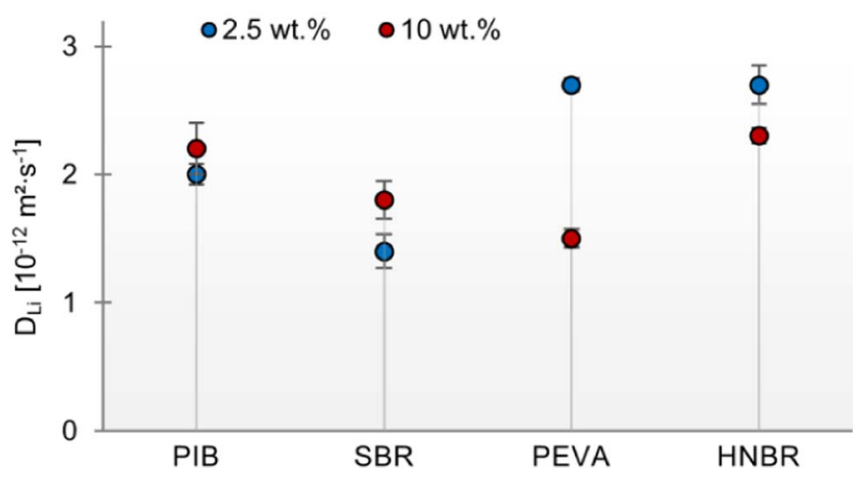

Figure 5. Li-ion diffusion coefficients for calendered SE sheets comprising 2.5 (blue) and $10 \mathrm{wt} \%$ (red) PIB, SBR, PEVA and HNBR, respectively, extracted from ${ }^{7} \mathrm{Li}$ PFG NMR measurements at $30^{\circ} \mathrm{C}$. Error bars indicate the measurement accuracy.

again be attributed to the described structural features of the polymer. Since roll-to-roll processes, which demand for a certain bendability, are very likely to be implemented for large-scale fabrication of ASSBs,${ }^{8}$ SBR-based layers might be unsuitable at this stage.

Evaluation of ionic conductivity.-Besides the mostly physical properties discussed above, the final and most important assessment criterion examined herein is the ionic conductivity of the SE layers. As mentioned, previous studies demonstrated that incorporation of a polymeric binder leads to a reduced ionic conductivity., ${ }^{4,9,14,19} \mathrm{~A}$ much stronger influence was found if the binder forms a film on the SE particles instead of granular domains in between the particles. ${ }^{28}$ Furthermore, it has been shown for various SE materials that conductivity increases with packing density, i.e. with decreasing porosity. ${ }^{17,31,32}$ Consequently, clear differences in ionic conductivity between the SE layers in this contribution are expected.

The long-range Li-ion transport was probed by means of ${ }^{7} \mathrm{Li} \mathrm{PFG}$ NMR spectroscopy of calendered SE sheets with different binder contents. As demonstrated by Kaus et al., ${ }^{24}$ this powerful technique allows for investigation of Li-ion diffusion on time scales of tens or hundreds of milliseconds, corresponding to length scales of several micrometers. An exemplary echo damping versus gradient strength plot can be found in Figure S4 for LSPS-PEVA. Table S1 shows the extracted diffusion coefficients $D_{L i}$ for SE sheets containing 2.5 and $10 \mathrm{wt} \%$ of the various binders, respectively. For better visualization, they are depicted in Figure 5 in blue $(2.5 \mathrm{wt} \%)$ and red (10 wt \%). At first glance it is clear that the impact of binder volume on lithium ion diffusivity depends on the binder used. For HNBR and PEVA, $D_{L i}$ decreases by roughly 15 and $44 \%$, respectively, with a fourfold increase in binder content. In contrast, it hardly changes for PIB, considering the measurement accuracy, and even rises by roughly $29 \%$ for SBR.

To better understand the differences, these results were connected with those from EDX analysis. The elemental maps indicated a continuous film of PEVA and HNBR on the SE particles. It is thus conceivable that a larger amount of binder, which leads to thicker polymer films, hinders Li-ion transfer between particles. On the contrary, due to the granular structure of PIB and SBR, the binder quantity has less impact on Li-ion diffusion. The enhanced bonding at larger binder volume indeed rather promotes $\mathrm{Li}$-ion transport at grain boundaries. For SBR, where poor adhesion requires a high binder content to yield freestanding sheets, this effect is more pronounced, resulting in a larger $D_{L i}$ value for the $10 \mathrm{wt} \%$ sample. This interpretation of the trends of $D_{L i}$ with increasing amount of binder is in line with the aforementioned findings of Inada et al., namely that a binder coating on SE particles strongly affects ionic transport, whereas granular binder domains have less impact. ${ }^{28}$ The influence of binder content though has not been studied in this context.
In order to assess the absolute values for $D_{L i}$ extracted from the PFG NMR measurements, they were first related to that of the pure LSPS powder, which was determined to $2.9 \pm 0.1 \cdot 10^{-12} \mathrm{~m}^{2} \cdot \mathrm{s}^{-1}$. Independent from the binder type and quantity, all SE sheets yielded lower values. In general, PEVA and HNBR outperform the others at low binder content, whilst PIB and HNBR show the highest diffusivity at high binder content. For a fair comparison, however, only those values corresponding to SE sheets that formed mechanically stable layers should be taken into account. As shown in Figure 3, the minimum binder content required to obtain processible, freestanding sheets varies from binder to binder. The absolute values for $2.5 \mathrm{wt} \%$ PIB and HNBR should thus be compared to that for $10 \mathrm{wt} \%$ SBR and PEVA. Accordingly, the highest diffusion coefficient of $2.7 \pm 0.15 \cdot 10^{-12} \mathrm{~m}^{2} \cdot \mathrm{s}^{-1}$ was yielded for HNBR, followed by PIB $\left(2.0 \pm 0.08 \cdot 10^{-12} \mathrm{~m}^{2} \cdot \mathrm{s}^{-1}\right)$, SBR $\left(1.8 \pm 0.15 \cdot 10^{-12} \mathrm{~m}^{2} \cdot \mathrm{s}^{-1}\right)$ and PEVA $\left(1.5 \pm 0.07 \cdot 10^{-12} \mathrm{~m}^{2} \cdot \mathrm{s}^{-1}\right)$. Compared to pure LSPS, addition of a polymeric binder resulted in a decrease of lithium ion diffusivity by $7 \%$ in the best and $48 \%$ in the worst case. This is in the same range as the $\sim 7-16 \%$ drop in lithium ion conductivity reported by Lee et al. for composite films of NBR $(4 \mathrm{wt} \%)$ and LPS vs. the pure material. ${ }^{18}$

The partial $\mathrm{Li}^{+}$conductivity $\sigma_{L i}$ can be derived from the diffusion coefficient $D_{L i}$ according to the Nernst-Einstein Equation 1. Here, $N_{L i}$ is the Li-ion concentration, $Q_{e}$ the unit charge, $k$ the Boltzmann constant and $T$ the temperature. In order to calculate the ionic conductivity, the Li-ion concentration has to be estimated first. This is usually done based on the crystal structure of the material, according to Equation 2. Here, $X_{L i}$ is the number of Li-ions per formula unit, $Z$ the number of formula units per unit cell and $V$ the volume of the unit cell.

$$
\begin{gathered}
\sigma_{\mathrm{Li}}=\frac{\mathrm{D}_{\mathrm{Li}} \cdot \mathrm{N}_{\mathrm{Li}} \cdot \mathrm{Q}_{\mathrm{e}}{ }^{2}}{\mathrm{k} \cdot \mathrm{T}} \\
\mathrm{N}_{\mathrm{Li}}=\frac{\mathrm{X}_{\mathrm{Li}} \cdot \mathrm{Z}}{\mathrm{V}}
\end{gathered}
$$

First, the conductivity of the pure SE material was calculated, using the NMR-derived diffusion coefficient $\left(2.9 \pm 0.1 \cdot 10^{-12}\right.$ $\left.\mathrm{m}^{2} \cdot \mathrm{s}^{-1}\right)$. The resulting ionic conductivity of $3.6 \pm 0.12 \mathrm{mS} \cdot \mathrm{cm}^{-1}$ is in line with literature data $\left(2-5 \mathrm{mS} \cdot \mathrm{cm}^{-1}\right)$, which were obtained through $\mathrm{AC}$ impedance measurements as well as different ${ }^{7} \mathrm{Li}$ NMR techniques. ${ }^{22,24,25}$ This shows that the Nernst-Einstein relationship allows for a good approximation of the ionic conductivity. For a meaningful assessment of the actual conductivity of the SE/binder composites, however, the lithium diffusion length in the NMR experiments has to be larger than the radius of the SE particles. Otherwise, $\mathrm{Li}$-ions would not cross grain-boundaries within the time span of the PFG NMR measurement, and the effect of the binder would thus be neglected.

A grain size of $250-300 \mathrm{~nm}$ is specified for LSPS by the supplier. ${ }^{33}$ In order to confirm this, X-ray diffraction (XRD) pattern of the LSPS powder used were recorded and refined with regard to grain size. The Rietveld refinement (see Figure S5) yielded an average crystallite size of roughly $148 \mathrm{~nm}$. The polymeric binder might, however, not reach each grain, but rather be located between particles. Based on the specific surface area of $1.6 \mathrm{~m}^{2} \cdot \mathrm{g}^{-1}$, determined by BET analysis, an average primary particle size of roughly $1 \mu \mathrm{m}$ can be estimated. ${ }^{34}$ This was supported by SEM imaging of the as-received LSPS powder (Figure S6). A diffusion length $d_{\text {diff }}>0.5 \mu \mathrm{m}$ would thus be desirable. It can be calculated as $\left(n \cdot D_{L i} \cdot t_{d i f f}\right)^{0.5}$ with $n$ being dimensionality of the motion and $t_{\text {diff }}$ the diffusion time. ${ }^{35}$ As LSPS is a $3 \mathrm{D}$ Li-ion conductor, ${ }^{36} n$ equals to 6 . The resulting $d_{\text {diff }}$ of roughly $1.3 \mu \mathrm{m}$ is much larger than the radius of the LSPS grains and still sufficiently larger than the radius of the primary particles.

Besides, two additional facts support the assumption that Li-ions are transported across grain-boundaries during the PFG NMR measurement. Firstly, the estimated ionic conductivity of the pure SE material matches the value reported for the total conductivity, while a significantly higher value would be expected for the intergrain 


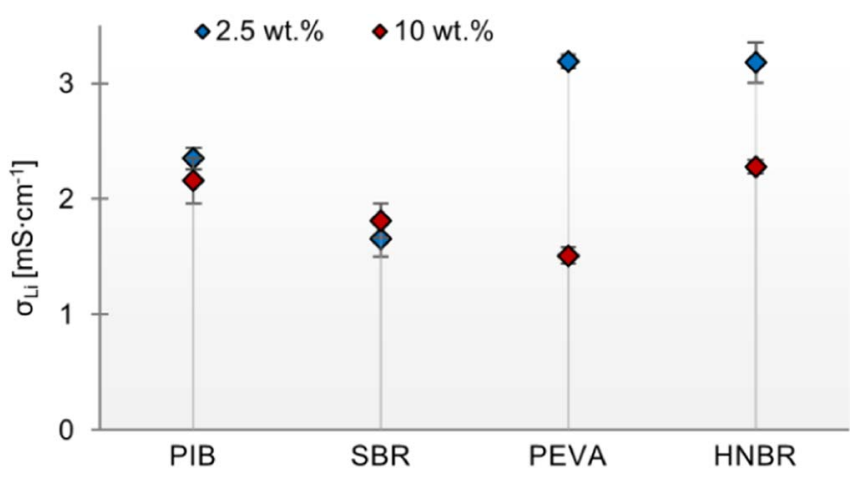

Figure 6. Estimated ionic conductivity for calendered SE sheets comprising $2.5 \mathrm{wt} \%$ (blue) and $10 \mathrm{wt} \%$ (red) PIB, SBR, PEVA and HNBR, respectively, at $30^{\circ} \mathrm{C}$. Error bars indicate the measurement accuracy, taking error propagation into account.

conductivity. ${ }^{22}$ Secondly, different values for $D_{L i}$ were extracted for the different binder types and volumes, all of them lower than that for pristine LSPS. If diffusion would solely take place within single SE particles, binder type and weight fraction would, however, not affect the measured diffusion coefficients. Hence, PFG NMR is suitable to probe the conductivity of the composite sheets without neglecting the binder impact. In order to account for the lithium-free volume though it is necessary to recalculate the $\mathrm{Li}$-ion concentration by integrating the volume fraction of the SE into Equation 2:

$$
\mathrm{N}_{\mathrm{Li}}^{\prime}=\left(100 \%-\mathrm{Vol} \%_{\text {Binder }}\right) \cdot \mathrm{N}_{\mathrm{Li}}
$$

The ionic conductivity can then be calculated using Equations 1 and 3. Figure 6 depicts the results for the different SE sheets containing 2.5 (blue) and $10 \mathrm{wt} \%$ (red) binder, respectively. A rise in conductivity with binder content is only observed for SBR. The increase by roughly $10 \%$ can again be attributed to the better adhesion between particles, resulting in a lower porosity around $30 \%$ for the $10 \mathrm{wt} \%$ compared to roughly $57 \%$ for the $2.5 \mathrm{wt} \%$ sheet. Apparently, poor adhesion is in this case more limiting than restricted ionic pathways due to blocking by greater amounts of polymeric binder. A similar observation has been made by Rosero-Navarro et al, who reported an increase in conductivity when incorporating ethyl cellulose in $\mathrm{Li}_{6} \mathrm{PS}_{5} \mathrm{Cl}$ pellet. ${ }^{14}$ In contrast, a fourfold binder content results in reduced conductivity for the other binders by roughly $10-50 \%$. Restriction of ionic pathways seems to be the limiting factor in the case of PIB, PEVA and HNBR. This is in line with the considerably lower porosity of the respective SE sheets compared to SBR (see Figure 3), which indicates that adhesion is not a major issue with these polymers.

As in the case of the diffusion coefficients, the required binder content to obtain mechanically stable layers should be considered when assessing the absolute values. Accordingly, the estimated ionic conductivity for $2.5 \mathrm{wt} \%$ PIB and HNBR should be compared to that for $10 \mathrm{wt} \%$ SBR and PEVA. The highest conductivity of $3.2 \pm 0.18 \mathrm{mS} \cdot \mathrm{cm}^{-1}$ was thus obtained for HNBR, followed by PIB $\left(2.4 \pm 0.09 \mathrm{mS} \cdot \mathrm{cm}^{-1}\right)$, SBR $\left(1.8 \pm 0.15 \mathrm{mS} \cdot \mathrm{cm}^{-1}\right)$ and PEVA $\left(1.5 \pm 0.07 \mathrm{mS} \cdot \mathrm{cm}^{-1}\right)$. In line with previous reports, stating that incorporation of a polymeric binder leads to a reduced ionic conductivity, ${ }^{4,7,9,14,19}$ all values level below that of pure LSPS $\left(3.6 \pm 0.12 \mathrm{mS} \cdot \mathrm{cm}^{-1}\right)$. The conductivity is reduced by roughly $12 \%$ in the best and $58 \%$ in the worst case. As mentioned, a comparable decrease of the ionic conductivity of composite films vs. the pure SE has been reported by Lee et al. ${ }^{18}$ They found that incorporation of $4 \mathrm{wt} \%$ NBR and butadiene, respectively, leads to a drop by roughly $7-27 \%$. When using $5.5 \mathrm{wt} \%$ NBR in a LPS sheet, Oh et al. maintained $\sim 50 \%$ of the ionic conductivity. ${ }^{19}$ Comparable results were shown by Rosero-Navarro et al. for a composite of $\mathrm{Li}_{6} \mathrm{PS}_{5} \mathrm{Cl}$ and ethyl cellulose. ${ }^{14}$ The group of Jung et al. demonstrated that even the application of a polymer scaffold that makes up $14 \mathrm{wt} \%$ lowers the conductivity only by a factor of roughly $3.6 .{ }^{20}$ Since all estimated ionic conductivities in this contribution lie in the $\mathrm{mS}$ range, the composite sheets prove to be suitable for application in ASSBs.

The respective ionic conductivity obtained through electrochemical impedance spectroscopy (EIS), however, might be lower since contacting issues are less pronounced in PFG NMR measurements. More precisely, PFG NMR only probes Li-ion diffusion between interconnected particles. The impact of porosity, however, is neglected. Additional effects specific to EIS measurements result from the interface between the SE sheet and the current collector. Pores as well as polymeric binder at the surface lead to a lower effective contacting area. As the measured resistance is normalized to the area, considering only the geometric dimensions of the sample, the conductivity is usually underestimated. Due to the high sensitivity of EIS to sample preparation and contacting, a more reliable comparison of the SE sheets with different binder types and volumes is achieved using PFG NMR. On the other hand, the EIS derived conductivity might be more relevant in an actual ASSB, where contacting issues are not negligible. In order to compare the two methods, an exemplary EIS measurement was performed of a LSPS sheet with $2.5 \mathrm{wt} \%$ HNBR (Figure S7). Fitting of the data yielded an ionic conductivity of roughly $0.3 \mathrm{mS} \cdot \mathrm{cm}^{-1}$, being an order of magnitude lower than that estimated from PFG NMR. Beside the aforementioned effects attributed to EIS, blocking of certain conduction pathways by a thick HNBR film might be responsible for the significant drop in total conductivity. This is again in line with the report from Inada et al., who observed that a continuous binder film covering SE particles remarkably hinders ionic transport. ${ }^{28}$ However, as stated by the group of Jung et al., ${ }^{19,37}$ an ionic conductivity in the $10^{-4} \mathrm{~S} \cdot \mathrm{cm}^{-1}$ range is still sufficiently high for application in ASSBs.

\section{Discussion}

In the present study we investigated five different polymers with regard to their suitability as binders for processing thin and freestanding SE layers with low porosity. All polymers fulfilled the basic requirements, namely solubility in toluene, negligible electronic conductivity and chemical stability in contact with the SE. Our extended slurry-processing study revealed that the optimal process parameters for the fabrication of SE sheets strongly depend on the used binder. Viscosity as well as binder content have to be adapted from case to case. Furthermore, some general trends could be identified. A correlation was found between the required minimum amount of binder and the molecular weight of the polymer. The higher the molecular weight, the less binder is needed. Number and type of functional groups attached to the polymer backbone are further determinants of the quality of the SE sheets. Cohesive forces between the slurry components compete with adhesive forces between slurry and carrier foil. Consequently, combination of a non-polar polymer backbone with a moderate amount of attached polar functional groups was found to be most effective. Bulky side groups, however, seem to hinder intense adhesion of the binder to the SE particles. This leads to larger quantities required for obtaining mechanically stable layers, as well as poorer bending properties of the sheets.

Considering our findings, the following properties should be pursued beyond the aforementioned basic requirements when searching for an ideal binder for sulfide-based SEs: (i) high molecular weight to minimize the required binder content, (ii) soft texture to improve densification properties, (iii) fully saturated hydrocarbon backbone to reduce stiffness and (iv) small polar functional groups (e.g. nitrile) to enhance interaction with SE particles without introducing steric hindrance. Among these characteristics, considered choice of proper side groups seems to be the most important one. As sulfur atoms are known to strongly interact with one another (sulfur bridge), an even better adhesion compared to HNBR might be achieved when using sulfur-containing polymers. A broad overview in this context has been provided by Goethals et al., covering information about chemical reactivity, softening properties, thermal stability and commercial availability. ${ }^{38}$ Diez et al. showed that electrically insulating sulfur copolymers can be adjusted with regard to mechanical and thermal 
properties. ${ }^{39}$ Successful application of sulfur-containing polymers has already been reported for $\mathrm{Li}-\mathrm{S}$ batteries ${ }^{40}$ as well as fuel cells. ${ }^{41}$ It would thus be worth testing their suitability as polymeric binders in SE layers for ASSBs in future studies.

The most important characteristic that determines whether a SE sheet is applicable in ASSBs is its ionic conductivity. We performed ${ }^{7} \mathrm{Li}$ PFG NMR measurements to study the impacts of binder type and content on conductivity. In general, all LSPS sheets yielded lower NMR-derived conductivities than the pure SE powder. The best results were obtained for those binders that formed freestanding sheets already at a low content of $2.5 \mathrm{wt} \%$. Apparently, the better the properties of the polymer, meaning high adhesive strength and high deformability, the lower is its influence on Li-ion diffusivity. Apart from that, the ionic conductivity is affected by binder quantity to a greater or lesser extent, depending on the distribution pattern of the binder. It seems that a polymer coating on the SE particles is more detrimental to Liion diffusion than granular binder domains between the SE particles. Generally speaking, a lower binder weight fraction results in a higher conductivity, as restriction of ionic pathways increases with binder content. This applies, however, only as long as sufficient polymer is present to ensure proper adhesion between the SE particles. Once limited adhesive properties become the restraining factor, conductivity will drop with decreasing binder content. For the most promising SE/binder composite with $2.5 \mathrm{wt} \% \mathrm{HNBR}$, the ionic conductivity of the composite sheet was also determined by impedance under blocking conditions. While the EIS-derived conductivity was roughly 10 fold lower $\left(\sim 0.3 \mathrm{mS} \cdot \mathrm{cm}^{-1}\right.$, see Figure S6) than the NMR-derived conductivity $\left(\sim 3.2 \mathrm{mS} \cdot \mathrm{cm}^{-1}\right.$, see Figure 6$)$, most likely caused by the effect of insulating polymer films in between the SE particles, a conductivity of $0.3 \mathrm{mS} \cdot \mathrm{cm}^{-1}$ would still be sufficiently high for application in ASSBs.

\section{Conclusions}

In summary, we showed that both the type and the amount of the used binder strongly affects the resulting properties of SE/polymer composite sheets with regards to homogeneity, porosity, mechanical stability and ionic conductivity. Among the tested polymers, HNBR showed the best results, followed by PIB and PEVA. Due to the large amount of binder required and the poor mechanical properties of the resulting SE sheet, SBR seems to be rather unsuitable as binder. PMMA can be completely excluded as processing was not possible. This study emphasizes the necessity of a careful selection of the binder for slurry-based processing of SE sheets. While a poor choice might lead to mechanically unstable layers, a well selected binder allows for fabrication of dense and flexible SE sheets with sufficiently high ionic conductivity to be applied in ASSBs. Although we could only investigate a limited number of polymers, the observed general trends should provide a guideline for binder selection beyond the materials employed herein. In future studies, the fabricated SE sheets will be tested in sheet-type ASSB cells to confirm their suitability.

\section{Acknowledgment}

The authors thank the BMW AG for the financial support of this work. Kathrin Moser and Nicolas Billot from the Institute for Machine Tools and Industrial Management $(i w b)$ of the TU München are greatly acknowledged for their support with the Mandrel Bend Tests. We thank Christian Sedlmeier from the Chair of Technical Electrochemistry (TEC) of the TU München for his support with the impedance measurements, using a measurement cell designed by Tanja Zünd and Johannes Landesfeind from TEC.
Sylvio Indris (iD https://orcid.org/0000-0002-5100-113X

Stefan J. Sedlmaier (D) https://orcid.org/0000-0002-5337-3076

\section{References}

1. D. Andre, H. Hain, P. Lamp, F. Maglia, and B. Stiaszny, J. Mater. Chem. A, 5(33), 17174 (2017).

2. F. Mizuno, C. Yada, and H. Iba, in Lithium-Ion Batteries, p. 273, Elsevier B.V. (2014).

3. J. Janek and W. G. Zeier, Nat. Energy, 1(9), 16141 (2016).

4. A. Sakuda, K. Kuratani, M. Yamamoto, M. Takahashi, T. Takeuchi, and H. Kobayashi, J. Electrochem. Soc., 164(12), A2474 (2017)

5. M. Yamamoto, M. Takahashi, Y. Terauchi, Y. Kobayashi, S. Ikeda, and A. Sakuda, J. Ceram. Soc. Japan, 125(5), 391 (2017).

6. K. Kerman, A. Luntz, V. Viswanathan, Y.-M. Chiang, and Z. Chen, J. Electrochem. Soc., 164(7), A1731 (2017)

7. Y. J. Nam, D. Y. Oh, S. H. Jung, and Y. S. Jung, J. Power Sources, 375, 93 (2018).

8. J. Schnell, T. Günther, T. Knoche, C. Vieider, L. Köhler, A. Just, M. Keller S. Passerini, and G. Reinhart, J. Power Sources, 382, 160 (2018).

9. M. Yamamoto, Y. Terauchi, A. Sakuda, and M. Takahashi, Sci. Rep., 8(1), 1212 (2018).

10. W. Weppner, in Encyclopedia of Electrochemical Power Sources, p. 162, Elsevier B.V. (2009)

11. C. Cao, Z.-B. Li, X.-L. Wang, X.-B. Zhao, and W.-Q. Han, Front. Energy Res., 2(Part 1), 947 (2014).

12. J. B. Goodenough and P. Singh, J. Electrochem. Soc., 162(14), A2387 (2015).

13. F. Han, T. Gao, Y. Zhu, K. J. Gaskell, and C. Wang, Adv. Mater, 27(23), 3473 (2015).

14. N. C. Rosero-Navarro, T. Kinoshita, A. Miura, M. Higuchi, and K. Tadanaga, Ionics, 23(6), 1619 (2017)

15. Y. Kato, S. Hori, T. Saito, K. Suzuki, M. Hirayama, A. Mitsui, M. Yonemura, H. Iba, and R. Kanno, Nat. Energy, 1(4), 16030 (2016).

16. N. Kamaya, K. Homma, Y. Yamakawa, M. Hirayama, R. Kanno, M. Yonemura, T. Kamiyama, Y. Kato, S. Hama, K. Kawamoto, and A. Mitsui, Nat. Mater, 10(9), $682(2011)$.

17. Y. Seino, T. Ota, K. Takada, A. Hayashi, and M. Tatsumisago, Energy Environ. Sci., 7(2), 627 (2014).

18. K. Lee, S. Kim, J. Park, S. H. Park, A. Coskun, D. S. Jung, W. Cho, and J. W. Choi, J. Electrochem. Soc., 164(9), A2075 (2017).

19. D. Y. Oh, D. H. Kim, S. H. Jung, J.-G. Han, N.-S. Choi, and Y. S. Jung, J. Mater Chem. A, 5(39), 20771 (2017).

20. Y. J. Nam, S.-J. Cho, D. Y. Oh, J.-M. Lim, S. Y. Kim, J. H. Song, Y.-G. Lee, S.-Y. Lee, and Y. S. Jung, Nano Lett., 15(5), 3317 (2015).

21. S. Ito, S. Fujiki, T. Yamada, Y. Aihara, Y. Park, T. Y. Kim, S.-W. Baek, J.-M. Lee, S. Doo, and N. Machida, J. Power Sources, 248, 943 (2014).

22. P. Bron, S. Johansson, K. Zick, Schmedt auf der Günne, Jörn S. Dehnen, and B. Roling, J. Am. Chem. Soc., 135(42), 15694 (2013).

23. S. P. Ong, Y. Mo, W. D. Richards, L. Miara, H. S. Lee, and G. Ceder, Energy Environ Sci., 6(1), 148 (2013).

24. M. Kaus, H. Stöffler, M. Yavuz, T. Zinkevich, M. Knapp, H. Ehrenberg, and S. Indris, J. Phys. Chem. C, 121(42), 23370 (2017).

25. I. Tarhouchi, V. Viallet, P. Vinatier, and M. Ménétrier, Solid State Ionics, 296, 18 (2016).

26. R. Kanno and M. Murayama, J. Electrochem. Soc., 148(7), A742 (2001).

27. International Chemical Society Cards (ICSC):, toluene 0078.

28. T. Inada, K. Takada, A. Kajiyama, M. Kouguchi, H. Sasaki, S. Kondo, M. Watanabe, M. Murayama, and R. Kanno, Solid State Ionics, 158(3-4), 275 (2003).

29. B.-R. Lee and E.-S. Oh, J. Phys. Chem. C, 117(9), 4404 (2013).

30. Y. J. Choi, D. H. Shin, S. H. Kim, S. C. Park, G. B. Cho, and K. W. Kim, Solid State Phenom., 124-126, 1007 (2007).

31. A. Sakuda, A. Hayashi, and M. Tatsumisago, Sci. Rep., 3, 2261 (2013).

32. E. C. Bucharsky, K. G. Schell, A. Hintennach, and M. J. Hoffmann, Solid State Ionics, 274, 77 (2015).

33. NEI Corporation, NANOMYTE ${ }^{\circledR}$ SSE-10 (LSPS) Specification Sheet, https://neicorporation.com/specs/SSE-10 Spec Sheet \%28LSPS\%29.pdf 2016

34. Y. Waseda and A. Muramatsu, Morphology Control of Materials and Nanoparticles: Advanced Materials Processing and Characterization, Berlin, Heidelberg, Springer 2004

35. W. S. Price, Concepts Magn. Reson., 9(5), 299 (1997).

36. D. A. Weber, A. Senyshyn, K. S. Weldert, S. Wenzel, W. Zhang, R. Kaiser, S. Berendts, J. Janek, and W. G. Zeier, Chem. Mater, 28(16), 5905 (2016).

37. K. H. Park, D. Y. Oh, Y. E. Choi, Y. J. Nam, L. Han, J.-Y. Kim, H. Xin, F. Lin, S. M. Oh, and Y. S. Jung, Adv. Mater, 28(9), 1874 (2016)

38. E. J. Goethals and J. Macromol, Sci., Part C: Polym. Rev., 2(1), 73 (1968).

39. S. Diez, A. Hoefling, P. Theato, and W. Pauer, Polymers, 9(12), 59 (2017).

40. X.-G. Yu, J.-Y. Xie, J. Yang, H.-J. Huang, K. Wang, and Z.-S. Wen, J. Electroanal. Chem., 573(1), 121 (2004).

41. J. S. Choi, A. K. Mishra, N. H. Kim, G. Shin, and J. H. Lee, J. Appl. Electrochem., 43(8), 749 (2013) 